International Journal of Applied Mathematics

Volume 29 No. $6 \quad 2016,641-653$

ISSN: 1311-1728 (printed version); ISSN: 1314-8060 (on-line version)

doi: http://dx.doi.org/10.12732/ijam.v29i6.1

\title{
MOMENTS OF GENERALIZED ORDER STATISTICS FROM NEW WEIBULL-PARETO DISTRIBUTION AND ITS CHARACTERIZATION
}

\author{
M.D. Izhar Khan ${ }^{1 \S}$, Mohd Akram Raza Khan ${ }^{2}$ \\ ${ }^{1}$ Department of Mathematics \\ Faculty of Science \\ Islamic University in Madinah \\ P.O. Box 170, Madinah, K. SAUDI ARABIA \\ ${ }^{2}$ Department of Statistics \& Operations Research \\ Aligarh Muslim University \\ Aligarh, 202002, INDIA
}

\begin{abstract}
In this paper, recurrence relations for single and product moments of generalized order statistics from New Weibull-Pareto Distribution (NWPD) have been derived. The results for ordinary order statistics and $k^{\text {th }}$ record values and are deduced from the relations derived. A characterization results of this distribution is also obtained. Further, means of order statistics are computed numerically for some selected values of parameters.
\end{abstract}

AMS Subject Classification: 62G30, 62E10

Key Words: generalized order statistics, order statistics, record values, single and product moments, recurrence relations, New Weibull-Paerto Distribution and characterization

Received: February 16, 2016

(c) 2016 Academic Publications

${ }^{\S}$ Correspondence author 


\section{Introduction}

A random variable $X$ is said to have a New Weibull-Pareto Distribution [9] if its probability density function $(p d f)$ is given by

$$
f(x)=\frac{\beta \delta}{\theta}\left(\frac{x}{\theta}\right)^{\beta-1} \exp ^{-\delta\left(\frac{x}{\theta}\right)^{\beta}}, \quad 0<x<\infty, \quad \beta>0, \quad \theta>0 \text { and } \delta>0 .
$$

and the cumulative distribution function $(c d f)$ is

$$
\bar{F}(x)=\exp ^{-\delta\left(\frac{x}{\theta}\right)^{\beta}}
$$

where

$$
\bar{F}(x)=1-F(x) .
$$

Therefore, in view of (1) and (2), we have

$$
\bar{F}(x)=\frac{\theta}{\beta \delta}\left(\frac{x}{\theta}\right)^{1-\beta} f(x) .
$$

The main properties of the New Weibull-Pareto Distribution as follows:

1. If $\beta=1$, the failure(or hazard) rate is constant.

2. If $\beta>1$, the failure(or hazard) rate is an increasing function of $x$.

3. If $\beta<1$, the failure(or hazard) rate is a decreasing function of $x$.

The stated above property makes the NWPD suitable for modeling system with constant failure rate, components that wears faster with time and components that wears slower with time. For more details on this distribution and it application see [9].

The concept of generalized order statistics (gos) has been introduced and extensively studied by [4].

Let $n \in N$ be a given integer and $\widetilde{m}=\left(m_{1}, m_{2}, \ldots, m_{n-1}\right) \in \Re^{n-1}, k \geq 1$ be the parameters such that $\gamma_{i}=k+n-i+\sum_{j=1}^{n-1} m_{j}>0$ for $1 \leq i \leq n-1$.

Then $X(1, n, \widetilde{m}, k), X(2, n, \widetilde{m}, k), \ldots, X(r, n, \widetilde{m}, k)$ are called gos from continuous population with the cummulative distribution function (cdf) $F(x)$ and the probability density function $(p d f) f(x)$, if their joint pdf has the form

$$
k\left(\prod_{j=1}^{n=1} \gamma_{j}\right)\left(\prod_{i=1}^{n=1}\left[1-F\left(x_{i}\right)\right]^{m_{i}} f\left(x_{i}\right)\right)\left[1-F\left(x_{n}\right)\right]^{k-1} f\left(x_{n}\right)
$$




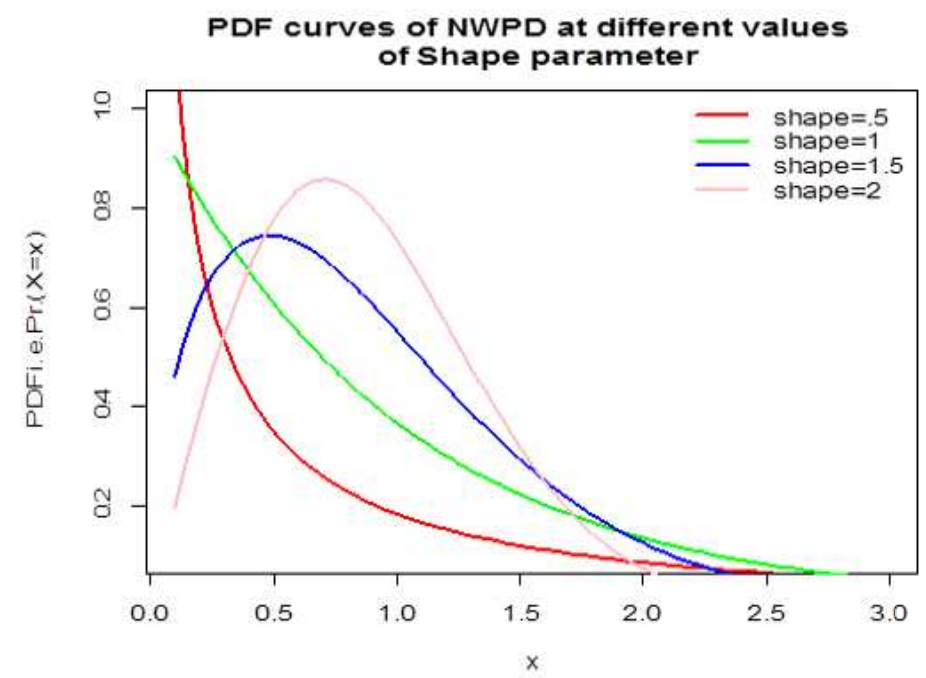

Figure 1: PDF curves of NWPD at different values of shape parameter.

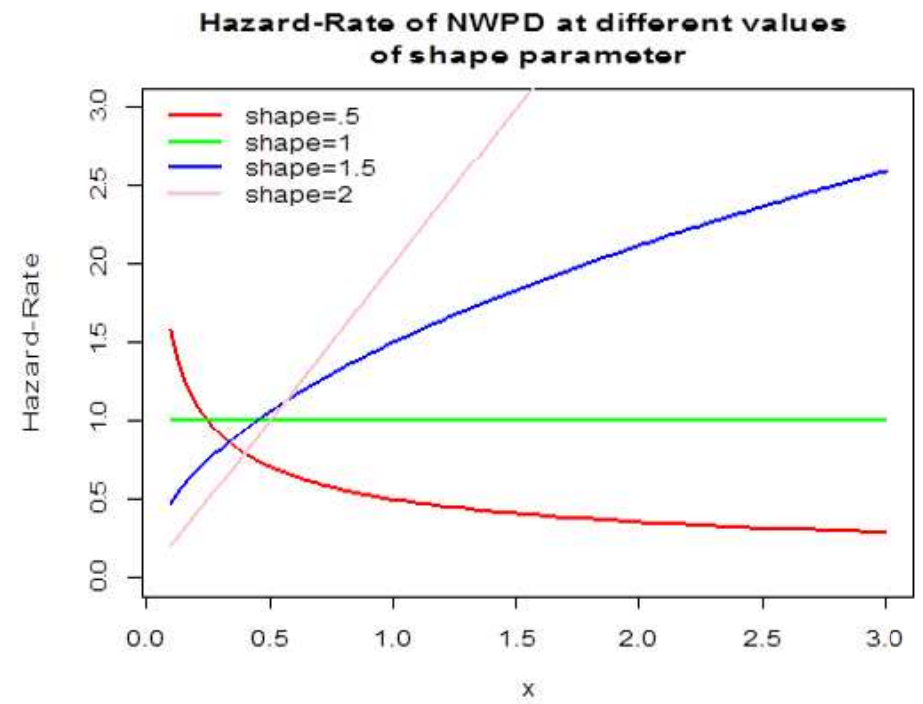

Figure 2: Hazard rates of NWPD at different values of shape parameter.

on the cone $F^{-1}(0+)<x_{1} \leq x_{2} \leq \ldots \leq x_{n}<F^{-1}(1)$ of $\Re^{n}$.

Choosing the parameters appropriately, models such as ordinary order statis- 


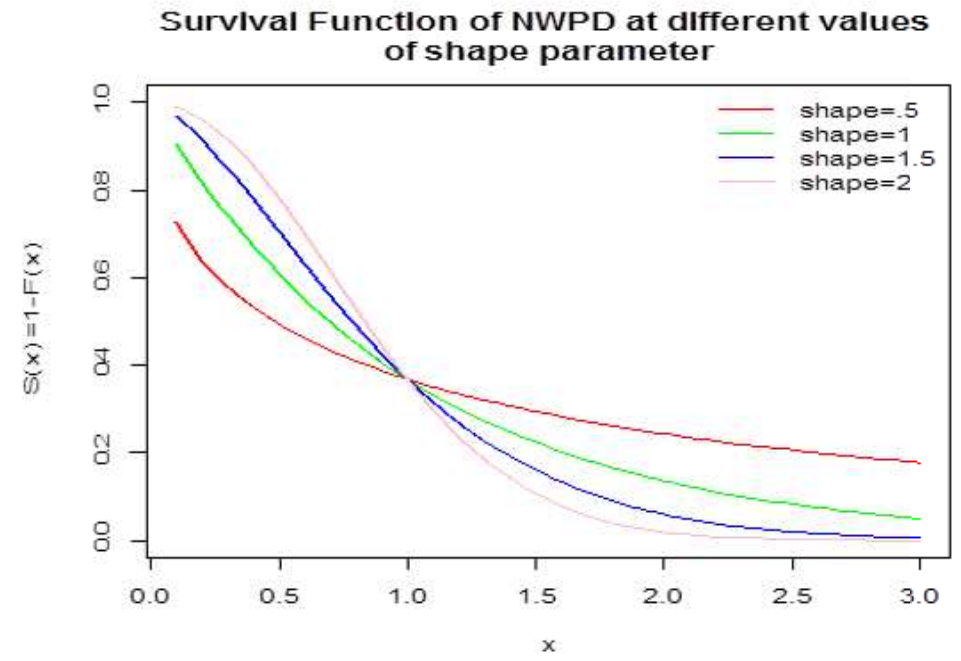

Figure 3: Survival function of NWPD at different values of shape parameter.

tics $\left(\gamma_{i}=n-i-1 ; i=1,2, \ldots, n\right.$, i.e $\left.m_{1}=m_{2}=m_{n-1}=0\right)$, $\mathrm{k}$-th record values $\left(\gamma_{i}=k\right.$, i.e. $\left.m_{1}=m_{2}=m_{n-1}=-1, k \in N\right)$, sequential order statistics $\left(\gamma_{i}=(n-i+1) \alpha_{i} ; \alpha_{1}, \alpha_{2}, \ldots, \alpha_{n}>0\right)$, order statistics with non-integral sample size $\left(\gamma_{i}=\alpha-i+1 ; \alpha>0\right)$, Pfefier's record values $\left(\gamma_{i}=\beta_{i} ; \beta_{1}, \beta_{2}, \ldots \beta_{n}>0\right)$ and progressive type II censored order statistics $\left(m_{i} \in N\right), k \in N$ are obtained by $[4]$.

For simplicity we shall assume $m_{1}=m_{2}=m_{n-1}=-m$.

The $p d f$ of $X(r, n, m, k)$ is

$$
f_{X(r, n, m, k)}(x)=\frac{C_{r-1}}{(r-1) !}[\bar{F}(x)]^{\gamma_{r}-1} \mathrm{~g}_{m}^{r-1}[F(x)] f(x)
$$

and the joint $p d f$ of $X(r, n, m, k)$ and $X(s, n, m, k), 1 \leq r<s \leq n$, is

$$
\begin{aligned}
& f_{x(r, n, m, k), x(s, n, m, k)}(x, y)=\frac{C_{s-1}}{(r-1) !(s-r-1) !}[\bar{F}(x)]^{m} \mathrm{~g}_{m}^{r-1}[F(x)] \\
& \times\left[h_{m}(F(y))-h_{m}(F(x))\right]^{s-r-1}[\bar{F}(y)]^{\gamma_{s}-1} f(x) f(y), \quad \alpha \leq x<y \leq \beta,
\end{aligned}
$$

where 


$$
\begin{gathered}
\gamma_{i}=k+(n-i)+\sum_{j=r}^{n-1} m_{j}=k+(n-i)+(m+1), \quad C_{r-1}=\prod_{i=1}^{r} \gamma_{i}, \\
h_{m}(x)= \begin{cases}-\frac{1}{m+1}(1-x)^{m+1} & m \neq-1 \\
-\log (1-x) & m=-1\end{cases}
\end{gathered}
$$

and

$$
\mathrm{g}_{m}(x)=h_{m}(x)-h_{m}(0), \quad x \in[0,1) .
$$

The result given in the paper can be used to compute the moments of ordered random variables, if the parent distribution follows the (NWPD), since the recurrence relations reduce the amount of direct computation and hence reduce the time and labour.

The recurrence relations based on generalized order statistics have obtained considerable attention in recent years. Many authors derived recurrence relations for generalized order statistics for different distributions, see [1], [2], [7], [5], [6] among others.

The paper is organized as follows. Section 2 presents a recurrence relation for single moment. Section 3 gives a recurrence relation for product moment. Section 4 is devoted to characterize the New Weibull-Pareto Distribution based on generalized order statistics. Section 5 delivers the numerical computations for some selected values of parameters, in context to the means of order statistics.

\section{Recurrence Relation for Single Moment}

Theorem 2.1. For the New Weibull-Pareto Distribution given (1) and $n \in N$, $m \in R, 2 \leq r \leq n$

$$
E\left[X^{j}(r, n, m, k)\right]-E\left[X^{j}(r-1, n, m, k)\right]=\frac{j \theta^{\beta}}{\gamma_{r} \beta \delta} E\left[X^{j-\beta}(r, n, m, k)\right] .
$$

Proof. From (5), we have

$$
E\left[X^{j}(r, n, m, k)\right]=\frac{C_{r-1}}{(r-1) !} \int_{0}^{\infty} x^{j}[\bar{F}(x)]^{\gamma_{r}-1} \mathrm{~g}_{m}^{r-1}[F(x)] f(x) d(x) .
$$

By performing integrating by part taking $[\bar{F}(x)]^{\gamma_{r}-1} f(x)$ as the part to be integrated, we get 


$$
\begin{aligned}
& E\left[X^{j}(r, n, m, k)\right]=E\left[X^{j}(r-1, n, m, k)\right] \\
& \quad+\frac{j C_{r-1}}{\gamma_{r}(r-1) !} \int_{0}^{\infty} x^{j-1}[\bar{F}(x)]^{\gamma_{r}} \mathrm{~g}_{m}^{r-1}[F(x)] d(x) .
\end{aligned}
$$

The constant of integration vanishes since the integral considered in (8) is a definite integral, on using (3), we obtain

$$
E\left[X^{j}(r, n, m, k)\right]-E\left[X^{j}(r-1, n, m, k)\right]=\frac{j \theta^{\beta}}{\gamma_{r} \beta \delta} E\left[X^{j-\beta}(r, n, m, k)\right]
$$

and hence the theorem follows.

Remark 2.1. Setting $m=0, k=1$ in Theorem 2.1, we obtain a recurrence relation for single moment of order statistics of the New Weibull-Pareto Distribution in the form

$$
E\left[X_{r: n}^{j}\right]-E\left[X_{r-1: n}^{j}\right]=\frac{j \theta^{\beta}}{(n-r+1) \beta \delta} E\left[X_{r: n}^{j-\beta}\right] .
$$

Remark 2.2. Setting $m=-1, k=1$ in Theorem 2.1, we get the recurrence relation for the single moment of $k^{t h}$ upper values of the New Weibull-Pareto Distribution in the form

$$
E\left[X_{U(r)}^{j}\right]^{k}-E\left[X_{U(r-1)}^{j}\right]^{k}=\frac{j \theta^{\beta}}{k \beta \delta} E\left[X_{U(r)}^{j-1}\right]^{k} .
$$

\section{Recurrence Relation for Product Moments}

Theorem 3.1. For the New Weibull-Pareto Distribution given (1) and $n \in N$, $m \in R, 1 \leq r \leq s \leq n-1$

$$
\begin{array}{r}
E\left[X^{i}(r, n, m, k) X^{j}(s, n, m, k)\right]-E\left[X^{i}(r, n, m, k) X^{j}(s-1, n, m, k)\right] \\
=\frac{j \theta^{\beta}}{\gamma_{s} \beta \delta} E\left[X^{i}(r, n, m, k) X^{j-\beta}(s, n, m, k)\right] .
\end{array}
$$

Proof. From (6), we have

$$
\begin{aligned}
& E\left[X^{i}(r, n, m, k) X^{j}(s, n, m, k)\right] \\
& \quad=\frac{C_{r-1}}{(r-1) !(s-r-1) !} \int_{0}^{\infty} x^{i}[\bar{F}(x)]^{m} \mathrm{~g}_{m}^{r-1}[F(x)] f(x) I(x) d(x),
\end{aligned}
$$


where

$$
I(x)=\int_{x}^{\infty} y^{j}[\bar{F}(y)]^{\gamma_{s}-1}\left[h_{m}(F(y))-h_{m}(F(x))\right]^{s-r-1} f(y) d y .
$$

Solving the integral in $I(x)$ by parts and substituting the resulting expression in (12), we get

$$
\begin{aligned}
& E\left[X^{i}(r, n, m, k) X^{j}(s, n, m, k)\right]-E\left[X^{i}(r, n, m, k) X^{j}(s-1, n, m, k)\right] \\
& =\frac{j C_{s-1}}{\gamma_{s}(r-1) !(s-r-1) !} \int_{0}^{\infty} \int_{x}^{\infty} x^{i} y^{j-1}[\bar{F}(x)]^{m} \mathrm{~g}_{m}^{r-1}[F(x)] f(x) \\
& \times\left[h_{m}(F(y))-h_{m}(F(x))\right]^{s-r-1}[F(y)]^{\gamma_{s}} d y d x .
\end{aligned}
$$

The constant of integration vanishes since the integral in $I(x)$ is a definite integral. On using relation (3), we obtain

$$
\begin{gathered}
E\left[X^{i}(r, n, m, k) X^{j}(s, n, m, k)\right]-E\left[X^{i}(r, n, m, k) X^{j}(s-1, n, m, k)\right] \\
=\frac{j C_{s-1} \theta^{\beta}}{\gamma_{s} \beta \delta(r-1) !(s-r-1) !} \int_{0}^{\infty} \int_{x}^{\infty} x^{i} y^{j-1}[\bar{F}(x)]^{m} \mathrm{~g}_{m}^{r-1}[F(x)] f(x) \\
\times\left[h_{m}(F(y))-h_{m}(F(x))\right]^{s-r-1}[F(y)]^{\gamma_{s}-1} f(y) d y d x . \\
E\left[X^{i}(r, n, m, k) X^{j}(s, n, m, k)\right]-E\left[X^{i}(r, n, m, k) X^{j}(s-1, n, m, k)\right] \\
=\frac{j \theta^{\beta}}{\gamma_{s} \beta \delta} E\left[X^{i}(r, n, m, k) X^{j-\beta}(s, n, m, k)\right],
\end{gathered}
$$

and hence the theorem follows.

Remark 3.1. Setting $m=0, k=1$ in Theorem 3.1, we obtain a recurrence relation for product moment of order statistics of the New Weibull-Pareto Distribution in the form

$$
E\left[X_{r, s: n}^{i j}\right]-E\left[X_{r, s-1: n}^{i j}\right]=\frac{j \theta^{\beta}}{(n-s+1) \beta \delta} E\left[X_{r, s: n}^{i j-\beta}\right] .
$$

Remark 3.2. Setting $m=-1, k=1$ in Theorem 3.1, we get the recurrence relation for the single moment of $k^{\text {th }}$ upper record values of the New WeibullPareto Distribution in the form

$$
E\left[X_{U(r)}^{i} X_{U(s)}^{j}\right]^{k}-E\left[X_{U(r)}^{i} X_{U(s-1)}^{j}\right]^{k}=\frac{j \theta^{\beta}}{k \beta \delta} E\left[X_{U(r)}^{i} X_{U(s)}^{j-\beta}\right]^{k} .
$$




\section{Characterizations}

This section discusses the characterization of NWPD. Characterization of a probability distribution plays an important role in probability and statistics. A probability distribution can be characterized through various method. In recent years, there has been a great interest in the characterizations of probability distributions through recurrence relations based on (gos).

Theorem 4.1 is based on the following result of [8], which is given below as Proposition 1.

Proposition 1. Let $n_{0}$ be any fixed non-negative integer and let $a, b$ be real numbers such that $-\infty<a<b<\infty$. Let $g(x) \geq 0$ be an absolutely continuous function with $g^{\prime}(x) \neq 0$ almost everywhere on $(a, b)$. Then the sequence of functions $\left\{[g(x)]^{n} \exp ^{-g(x)}, n \geq n_{0}\right\}$ is complete in $L(a, b)$ if and only if $g(x)$ is strictly monotone on $(a, b)$.

Using the above proposition, we get the following stronger version of Theorem 2.1.

Theorem 4.1. Let $X$ be a non-negative random variable having absolutely continuous distribution $F(x)$ with $F(0)=0$ and $0<F(x)<1$, for all $x>0$

$$
E\left[X^{j}(r, n, m, k)\right]=E\left[X^{j}(r-1, n, m, k)\right]+\frac{j \theta^{\beta}}{\gamma_{r} \beta \delta} E\left[X^{j-\beta}(r, n, m, k)\right]
$$

if and only if

$$
F(x)=1-\exp ^{-\delta\left(\frac{x}{\theta}\right)^{\beta}} \quad 0<x<\infty, \quad \beta>0, \quad \theta>0 \text { and } \delta>0 .
$$

Proof. The necessary part follows immediately from equation (7). On the other hand if the recurrence relation in equation (15) is satisfied, then

$$
\begin{gathered}
\frac{j \theta^{\beta}}{\gamma_{r} \beta \delta} E\left[X^{j-\beta}(r, n, m, k)\right]=E\left[X^{j}(r, n, m, k)\right]-E\left[X^{j}(r-1, n, m, k)\right] \\
\times \frac{j C_{r-1} \theta^{\beta}}{\gamma_{r} \beta \delta(r-1) !} \int_{0}^{\infty} x^{j-\beta}[\bar{F}(x)]^{\gamma_{r}-1} \mathrm{~g}_{m}^{r-1}[F(x)] f(x) d(x) \\
=\frac{C_{r-1}}{(r-1) !} \int_{0}^{\infty} x^{j}[\bar{F}(x)]^{\gamma_{r}-1} \mathrm{~g}_{m}^{r-1}[F(x)] f(x) d(x) \\
-\frac{C_{r-1}(r-1)}{\gamma_{r}(r-1) !} \int_{0}^{\infty} x^{j}[\bar{F}(x)]^{\gamma_{r}+m} \mathrm{~g}_{m}^{r-2}[F(x)] f(x) d(x)
\end{gathered}
$$




$$
=\frac{C_{r-1}}{(r-1) !} \int_{0}^{\infty} x^{j}[\bar{F}(x)]^{\gamma_{r}} \mathrm{~g}_{m}^{r-2}[F(x)] f(x)\left[\frac{\mathrm{g}_{m}[F(x)]}{[\bar{F}(x)]}-\frac{(r-1)[\bar{F}(x)]}{\gamma_{r}}\right] d(x) .
$$

Let

$$
\begin{aligned}
h(x) & =-\frac{[\bar{F}(x)]^{\gamma_{r}} \mathrm{~g}_{m}^{r-1}[F(x)]}{\gamma_{r}}, \\
h^{\prime}(x) & =[\bar{F}(x)]^{\gamma_{r}} \mathrm{~g}_{m}^{r-2}[F(x)] f(x)\left[\frac{\mathrm{g}_{m}[F(x)]}{[\bar{F}(x)]}-\frac{(r-1)[\bar{F}(x)]^{m}}{\gamma_{r}}\right] .
\end{aligned}
$$

Thus,

$$
\begin{aligned}
\frac{j C_{r-1} \theta^{\beta}}{\gamma_{r} \beta \delta(r-1) !} \int_{0}^{\infty} x^{j-\beta}[\bar{F}(x)]^{\gamma_{r}-1} \mathrm{~g}_{m}^{r-1}[F(x)] f(x) d(x) \\
=\frac{C_{r-1}}{(r-1) !} \int_{0}^{\infty} x^{j} h^{\prime}(x) d(x) .
\end{aligned}
$$

Integrating the RHS of (18) by part and using the value of $h(x)$, we get

$$
\begin{aligned}
\frac{j C_{r-1} \theta^{\beta}}{\gamma_{r} \beta \delta(r-1) !} \int_{0}^{\infty} x^{j-\beta}[\bar{F}(x)]^{\gamma_{r}-1} \mathrm{~g}_{m}^{r-1}[F(x)] f(x) d(x) \\
=\frac{C_{r-1}}{\gamma_{r}(r-1) !} \int_{0}^{\infty} j x^{j-1}[\bar{F}(x)]^{\gamma_{r}} \mathrm{~g}_{m}^{r-1}[F(x)] d(x),
\end{aligned}
$$

which is reduces to

$$
\frac{C_{r-1}}{(r-1) !} \int_{0}^{\infty} x^{j-1}[\bar{F}(x)]^{\gamma_{r}} \mathrm{~g}_{m}^{r-1}[F(x)]\left[\frac{\theta^{\beta}}{\beta \delta} \frac{f(x)}{\bar{F}(x)} x^{1-\beta}-1\right] d x=0 .
$$

It now follows form the above proposition, i.e.

$$
f(x)=\frac{\beta \delta}{\theta}\left(\frac{x}{\theta}\right)^{\beta-1} \bar{F}(x),
$$

which proves that $f(x)$ has the form as in (1).

The next Theorem 4.2 is a characterization based on the moments of minimal order statistics. Putting $n=1$ in (15).

Theorem 4.2. Let $j$ be a non-negative integer. A necessary and sufficient condition for a random variable $X$ to be distributed with pdf given by (1) is that,

$$
\frac{j \theta^{\beta}}{n \beta \delta} E\left[X_{1: n}^{j-\beta}\right]=E\left[X_{1: n}^{j}\right]
$$

Proof. The necessary part follows immediately from (7). On the other hand if the recurrence relation (20) is satisfied, then 


$$
\frac{j \theta^{\beta}}{n \beta \delta} \int_{0}^{\infty} x^{j-\beta}[\bar{F}(x)]^{n-1} f(x) d(x)=\int_{0}^{\infty} x^{j}[\bar{F}(x)]^{n-1} f(x) d(x) .
$$

Integrating the integrals on the right-hand side of the above expression by parts, we get

$$
\frac{j \theta^{\beta}}{n \beta \delta} \int_{0}^{\infty} x^{j-\beta}[\bar{F}(x)]^{n-1} f(x) d(x)=\frac{1}{n} \int_{0}^{\infty} j x^{j-1}[\bar{F}(x)]^{n} d(x)
$$

which further reduces to

$$
\int_{0}^{\infty} x^{j-1}[\bar{F}(x)]^{n-1}\left[\frac{\theta^{\beta}}{\beta \delta} f(x) x^{1-\beta}-\bar{F}(x)\right] d x=0, \quad n=1,2, \ldots .
$$

Now applying a generalization of the Müntz-Szász theorem [8] to equation (21), we obtain

$$
f(x)=\frac{\beta \delta}{\theta}\left(\frac{x}{\theta}\right)^{\beta-1} \bar{F}(x),
$$

which proves that $f(x)$ has the form as in (1).

\section{Numerical Computations}

By solving the equation (9) numerically, the mean of order statistics are computed for some selected values of the parameters.

These are provided in Table 1 and Table 2. 


\begin{tabular}{|c|c|c|c|c|c|}
\hline$n$ & $r$ & $\begin{array}{l}\theta=0.25, \\
\beta=1, \\
\delta=1\end{array}$ & $\begin{array}{l}\theta=0.50, \\
\beta=1, \\
\delta=1\end{array}$ & $\begin{array}{l}\theta=0.75, \\
\beta=1, \\
\delta=1\end{array}$ & $\begin{array}{l}\theta=1, \\
\beta=1, \\
\delta=1\end{array}$ \\
\hline 1 & 1 & 0.2500 & 0.5000 & 0.7500 & 1.0000 \\
\hline 2 & 1 & 0.1250 & 0.2500 & 0.3750 & 0.5000 \\
& 2 & 0.3750 & 0.7500 & 1.1250 & 1.5000 \\
\hline 3 & 1 & 0.0833 & 0.1670 & 0.2500 & 0.3300 \\
& 2 & 0.2083 & 0.4170 & 0.6250 & 0.8300 \\
& 3 & 0.4583 & 0.9170 & 1.3750 & 1.8300 \\
\hline 4 & 1 & 0.0625 & 0.1250 & 0.1875 & 0.2500 \\
& 2 & 0.1458 & 0.2920 & 0.4375 & 0.5800 \\
& 3 & 0.2708 & 0.5420 & 0.8125 & 1.0800 \\
& 4 & 0.5208 & 1.0420 & 1.5625 & 2.0800 \\
\hline 5 & 1 & 0.0500 & 0.1000 & 0.1500 & 0.2000 \\
& 2 & 0.1125 & 0.2250 & 0.3375 & 0.4500 \\
& 3 & 0.1958 & 0.3920 & 0.5875 & 0.7800 \\
& 4 & 0.3208 & 0.6420 & 0.9625 & 1.2000 \\
& 5 & 0.5708 & 1.1420 & 1.7125 & 2.2800 \\
\hline & \multicolumn{5}{|c}{} \\
\hline
\end{tabular}

Table 1: Means of order statistics from the New Weibull-Pareto distribution (NWPD). 


\begin{tabular}{|c|c|c|c|c|c|}
\hline$n$ & $r$ & $\begin{array}{l}\theta=0.25 \\
\beta=1 \\
\delta=2\end{array}$ & $\begin{array}{l}\theta=0.50 \\
\beta=1 \\
\delta=2\end{array}$ & $\begin{array}{l}\theta=0.75, \\
\beta=1, \\
\delta=2\end{array}$ & $\begin{array}{l}\theta=1, \\
\beta=1, \\
\delta=2\end{array}$ \\
\hline 1 & 1 & 0.1250 & 0.2500 & 0.3750 & 0.5000 \\
\hline \multirow[t]{2}{*}{2} & 1 & 0.0625 & 0.1250 & 0.1875 & 0.2500 \\
\hline & 2 & 0.1875 & 0.3750 & 0.5625 & 0.7500 \\
\hline \multirow[t]{3}{*}{3} & 1 & 0.0416 & 0.0830 & 0.1250 & 0.1667 \\
\hline & 2 & 0.1041 & 0.2080 & 0.3125 & 0.4167 \\
\hline & 3 & 0.2291 & 0.4580 & 0.6875 & 0.9167 \\
\hline \multirow[t]{4}{*}{4} & 1 & 0.0312 & 0.0625 & 0.0937 & 0.1250 \\
\hline & 2 & 0.0729 & 0.1455 & 0.2187 & 0.2917 \\
\hline & 3 & 0.1354 & 0.2705 & 0.4062 & 0.5417 \\
\hline & 4 & 0.2604 & 0.5205 & 0.7812 & 1.0417 \\
\hline \multirow[t]{5}{*}{5} & 1 & 0.0250 & 0.0500 & 0.0750 & 0.1000 \\
\hline & 2 & 0.0562 & 0.1125 & 0.1687 & 0.2250 \\
\hline & 3 & 0.0979 & 0.1955 & 0.2937 & 0.3917 \\
\hline & 4 & 0.1604 & 0.3205 & 0.4812 & 0.6417 \\
\hline & 5 & 0.2854 & 0.5705 & 0.8562 & 1.1417 \\
\hline
\end{tabular}

Table 2: Means of order statistics from the New Weibull-Pareto distribution (NWPD). 


\section{References}

[1] A.A. Ahmad and M. Fawzy, Recurrence relations for single and product moments of generalized order statistics from doubly truncated distributions, J. Stat. Plann. Infer., 17, No 2 (2002), 241-249.

[2] E.K. AL-Hussaini, A.A. M. Ahmad and M.A. AL-Kashif, Recurrence relations for moment and conditional moment genereating functions of generalized order statistics, Metrika, 61 (2005), 199-220.

[3] J.S. Hwang and G.D. Lin, On a generalized moments problem II, Proc. Amer. Math. Soc., 91 (1984), 577-580.

[4] U. Kamps, A Concept of Generalized Order Statistics. Teubner, Stuttgart (1995).

[5] M.I. Khan, M.A. R. Khan and M.A. Khan, Generalized order statistics from $q$-exponential type-1 distribution and its characterization. Int. J. Comp. Theo. Stat., 2, No 1 (2015), 1-6.

[6] M.I. Khan, M.A. R. Khan and M.A. Khan, Generalized order statistics from $q$-exponential type-II distribution and its characterization. J. Math. Comput. Sci., 5, No 4 (2015), 507-516.

[7] D. Kumar and M.I. Khan, Relations for generalized order statistics from doubly truncated generalized exponential distribution and its characterization, Math. Sci. Lett., 2, No 1 (2013), 9-18.

[8] G.D. Lin, On a moment problem, Tohoku Math. J., 38 (1986), 595-598.

[9] N. Suleman and L. Albert, The New Weibull-Pareto Distribution, Pak. J. Stat. Oper. Res., 11, No 1 (2015), 103-114. 
